\title{
Pattern of Recurrence of Glioblastoma Versus Grade 4 IDH-Mutant Astrocytoma Following Chemoradiation
}

\section{James Stewart}

Sunnybrook Research Institute

\section{Arjun Sahgal}

Odette Cancer Centre

Aimee K.M. Chan

Sunnybrook Health Sciences Centre

Hany Soliman

Odette Cancer Centre

Chia-Lin Tseng

Odette Cancer Centre

Jay Detsky

Odette Cancer Centre

\section{Sten Myrehaug}

Odette Cancer Centre

\section{Eshetu G Atenafu}

University Health Network
Ali Helmi
University of Toronto
James Perry

Sunnybrook Health Sciences Centre

Julia Keith

Sunnybrook Health Sciences Centre

Mary Jane Lim-Fat

Sunnybrook Health Sciences Centre

\section{David G Munoz}

Saint Michael's Hospital

\section{Gelareh Zadeh}

University Health Network

\section{David B. Shultz}

University Health Network

\section{Sunit Das}


Saint Michael's Hospital

\section{Catherine Coolens}

University Health Network

\section{Paula Alcaide-Leon}

University Health Network

Pejman Jabehdar Maralani ( Pejman.Maralani@sunnybrook.ca )

University of Toronto https://orcid.org/0000-0001-9975-4379

\section{Research Article}

Keywords: Glioblastoma, grade 4 IDH-mutant astrocytoma, recurrence, isocitrate dehydrogenase (IDH)

Posted Date: November 17th, 2021

DOl: https://doi.org/10.21203/rs.3.rs-1079782/v1

License: (c) (i) This work is licensed under a Creative Commons Attribution 4.0 International License. Read Full License 


\section{Abstract \\ Purpose}

To quantitatively compare the recurrence pattern of glioblastoma (IDH-wild type) versus grade 4 IDHmutant astrocytoma (herein referred to as wtIDH and mutIDH, respectively) following primary chemoradiation.

\section{Methods}

Twenty-two wtIDH and 22 mutIDH patients matched by sex, extent of resection, and corpus callosum involvement were enrolled. The recurrent gross tumor volume ( $\mathrm{rGTV}$ ) was compared with both the gross tumor volume (GTV) and clinical target volume (CTV) from radiotherapy planning. Failure patterns were quantified by the incidence and volume of the rGTV outside the GTV and CTV, and positional differences of the rGTV centroid from the GTV and CTV.

\section{Results}

The GTV was smaller in wtIDH compared to the mutIDH group (mean \pm SD: $46.5 \pm 26.0 \mathrm{~cm}^{3}$ v. $72.2 \pm 45.4$ $\mathrm{cm}^{3}, \mathrm{p}=0.026$ ). The rGTV was $10.7 \pm 26.9 \mathrm{~cm}^{3}$ and $46.9 \pm 55.0 \mathrm{~cm}^{3}$ smaller than the GTV for the same groups ( $p=0.018)$. The rGTV extended outside the GTV in $22(100 \%)$ and $15(68 \%)(p=0.009)$ of wtIDH and mutIDH patients, respectively; however, the volume of rGTV outside the GTV was not significantly different $\left(12.4 \pm 16.1 \mathrm{~cm}^{3}\right.$ vs. $\left.8.4 \pm 14.2 \mathrm{~cm}^{3}, \mathrm{p}=0.443\right)$. The rGTV metrics extending outside the CTV was not different between the groups. The rGTV centroid was within $5.7 \mathrm{~mm}$ of the closest GTV edge for 21 (95\%) and $22(100 \%)$ of wtIDH and mutIDH patients, respectively.

\section{Conclusion}

The rGTV extended beyond the GTV less often in mutIDH patients, suggesting limited margin radiotherapy could be beneficial in this group. The results support the study of small margin adaptive radiotherapy per the ongoing UNITED MR-Linac $5 \mathrm{~mm}$ CTV trial (NCT04726397).

\section{Introduction}

Isocitrate dehydrogenase (IDH) mutation status has established itself as a fundamental molecular biomarker in gliomas [1, 2] and serves as a defining feature in the 2016 and 2021 World Health Organization (WHO) classifications of brain tumors [3,4]. Patients with grade 4 IDH mutant astrocytoma [4] (previously referred to as IDH mutant glioblastoma [3]) have a demonstrated increase in progression free and overall survival compared to patients with glioblastoma [4] (previously referred to as IDH wild 
type glioblastoma [3]) [5-10]. The current standard of care for both de novo glioblastoma and grade 4 IDH-mutant astrocytoma (these two groups are hereafter referred to as wtIDH and mutIDH, respectively) is maximal safe surgical resection, followed by radiotherapy to 54-60 Gy in 30 fractions with concurrent followed by adjuvant temozolomide chemotherapy $[11,12]$.

Within this treatment strategy, patterns of failure with respect to the high dose radiation fields have been well characterized [13-16]. Such results have established the current radiation planning approach of targeting the surgical cavity and any residual enhancing tumor on T1-weighted MRI plus a $1.5-3 \mathrm{~cm}$ margin to include the region at the highest risk of recurrence [11, 17-19]. Despite differences in the underlying biology [20], the same radiotherapy guidelines are used for both wtIDH and mutIDH partially due to the lack of data on the relationship between IDH status and failure patterns.

The goal of this retrospective study was to quantitatively evaluate the pattern of recurrence between the wtIDH and mutIDH groups by assessing the volume of the recurrent tumor relative to upfront gross tumor and clinical target volumes from radiation planning. We also assessed the displacement of the centroid of the recurrent tumor relative to the radiotherapy planning volumes. Our hypothesis is that, given their underlying biologic differences, the tumor recurrence pattern differs between wtIDH and mutIDH following standard primary chemoradiotherapy.

\section{Methods And Materials}

\section{Patient characteristics, treatment, and imaging}

This retrospective study was approved by the research ethics board with waiver of the informed consent. Using our local database from 2009 to 2019, 22 consecutive adult (18 years old or older) patients with de novo grade 4 IDH-mutant astrocytoma (mutIDH group) who completed primary radiation (54-60 Gy in 30 fractions) with concurrent temozolomide chemotherapy were identified. Subsequently, 22 adult patients with de novo glioblastoma (wtIDH group) who also completed primary radiation (54-60 Gy in 30 fractions) with concurrent temozolomide chemotherapy were selected by matching to sex, extent of resection (gross total, subtotal, or biopsy), and involvement of corpus callosum. Matching by age was not feasible due to the generally younger age of mutIDH patients [21] and the resulting lack of age-matched wtIDH controls in our database. Patient characteristics are summarized in Table 1. 
Table 1

Patient Characteristics

\begin{tabular}{|llll|}
\hline & $\begin{array}{l}\text { wtIDH } \\
(\mathbf{n = 2 2})\end{array}$ & $\begin{array}{l}\text { mutlDH } \\
(\mathbf{n}=\mathbf{2 2})\end{array}$ & p-value \\
\hline Median Age in Years (Range) & $54.5(39-68)$ & $41.5(24-82)$ & $<0.001$ \\
\hline Sex & $15(68 \%)$ & $16(73 \%)$ & 1.00 \\
Male & $7(32 \%)$ & $6(27 \%)$ & \\
Female & & & \\
Resection Type & $4(18 \%)$ & $4(18 \%)$ & 1.00 \\
Gross Total & $17(77 \%)$ & $17(77 \%)$ & \\
Subtotal & $1(5 \%)$ & $1(5 \%)$ & \\
Biopsy & & & \\
Corpus Callosum Involvement & $12(55 \%)$ & $12(55 \%)$ & 1.00 \\
Yes & $10(45 \%)$ & $10(45 \%)$ & \\
No & & & \\
\hline
\end{tabular}

The gross tumor volume (GTV) and clinical target volume (CTV) contours from the radiation planning CT or MRI along with the gadolinium enhanced T1-weighted sequence from the MRI on which the recurrence was declared according to the RANO criteria [22] were downloaded to an offline workstation. The CTV and GTV were contoured in accordance with published guidelines $[23,24]$. The recurrent MRI was coregistered and resampled to the space of the planning CT or MRI using SPM12 (RRID:SCR_007037). The recurrent GTV (rGTV) was then manually contoured on the recurrent MRI as the T1 enhancing tumor by a neuroradiologist (PJM) using ITK-SNAP (RRID:SCR_002010) [25]. The rGTV contours were confirmed by a radiation oncologist specializing in the central nervous system (AS). All three contours analyzed in this work, along with the planned dose distribution, are illustrated for a single patient in Figure 1(a).

\section{Survival analysis}

Progression free and overall survival were assessed from the date of the planning CT or MRI with the resulting progression free survival (PFS) and overall survival (OS) rates calculated using the Kaplan-Meier product-limit method. The log-rank test was used to assess statistical significance of IDH mutation status on PFS and OS.

\section{Volumetric and positional analyses}

The GTV, CTV, and rGTV contours were exported as binary masks in the Analyze 7.5 image format. Positional and volumetric analyses was performed after importing these masks into MATLAB (v. 9.7.0; 
RRID:SCR_001622). The following metrics, illustrated in Figure 1(b), were quantified for each patient using an in-house script:

1. Volume of the GTV, CTV, and rGTV.

2. Volume of the rGTV outside of the GTV (the region labelled 'A' in Figure $1(b)$ ) and CTV (region ' $B$ ' in Figure $1(\mathrm{~b})$ ). This volume is presented as both an absolute volume and relative to the full rGTV volume.

3. Distance of the rGTV centroid from each of the GTV and CTV centroids, where the centroid is the geometric center of the three-dimensional contour. These are denoted by the arrows connecting the rGTV centroid (orange) to the GTV (red) and CTV (blue) centroids, respectively, in Figure 1(b).

4. Distance of the rGTV centroid to the closest edge of the GTV, illustrated by the arrow connecting the rGTV centroid (orange) to the GTV border (red) in Figure 1(b). If the rGTV centroid is within the GTV, this distance is zero.

\section{Statistical analysis}

Recurrence categorization outside the GTV and/or CTV was statistically assessed with Fisher's exact test. Student's paired t-test was used to compare the contour volumes, volumetric data, and positional data between the wtIDH and mutIDH groups. In all analyses, $p$-values were two-sided and $p<0.05$ was considered statistically significant. All statistical analyses were performed using the SAS software suite (v 9.4; RRID:SCR_008567).

\section{Results}

Patient characteristics are summarized in Table 1 demonstrating matching in terms of sex, extent of resection and involvement of corpus callosum between the wtIDH and mutIDH groups.

\section{Survival analysis}

Kaplan-Meier estimates of PFS and OS are outlined in Figure 2. Median PFS was 9.0 (95\% confidence interval (Cl): 4.9-12.9) and 15.1 months (95\% Cl: 6.0-28.6) for the wtIDH and mutIDH groups $(p=0.016)$, respectively. Similarly, median OS was 14.2 (95\% Cl: 10.1-19.2) and 42.2 months (95\% Cl, 23.8-50.2) for the same groups $(p<0.001)$.

\section{Volumetric analysis of recurrence}

Volumes of the GTV, CTV, and rGTV are summarized in Figure 3(a)-(c). The mean GTV was smaller in the wtIDH versus the mutIDH group (mean \pm SD: $46.5 \pm 26.0 \mathrm{~cm}^{3}$ versus $72.2 \pm 45.4 \mathrm{~cm}^{3}, p=0.026$ ). The CTV and rGTV volumes were not statistically different between wtIDH and mutIDH groups $(p=0.545$ and $p=0.308$, respectively). The difference between GTV and rGTV volumes in individual patients is 
illustrated in Figure 3(d)-(e); the rGTV was on average $10.7 \pm 26.9 \mathrm{~cm}^{3}$ smaller than the original GTV for the wtIDH group, and $46.9 \pm 55.0 \mathrm{~cm}^{3}$ smaller for mutIDH group $(p=0.018)$.

The volume of the rGTV outside the planning contours is summarized in Figure 4(a). For the wtIDH and mutIDH groups, the mean volume of the rGTV outside the GTV was $12.4 \pm 16.1 \mathrm{~cm}^{3}(26.8 \pm 22.4 \%$ of the rGTV) and $8.4 \pm 14.2 \mathrm{~cm}^{3}(28.4 \pm 29.5 \%)(p=0.443)$. The rGTV extended outside the GTV in $22(100 \%)$ and $15(68 \%)$ of wtIDH and mutIDH groups, respectively, as measured by the number of patients having greater than $0.3 \mathrm{~cm}^{3}$ of the rGTV outside the GTV $(p=0.009)$. The $0.3 \mathrm{~cm}^{3}$ threshold was used to account for small spurious regions where the rGTV and GTV share a border (e.g. along the dura). The rGTV extended outside the CTV in $6(27 \%)$ and $7(32 \%)$ patients from the same groups $(p=1.00)$. Finally, the mean rGTV volume outside the CTV was $2.7 \pm 11.0 \mathrm{~cm}^{3}\left(5.2 \pm 20.5 \%\right.$ of the rGTV) and $0.6 \pm 1.2 \mathrm{~cm}^{3}(4.5$ $\pm 7.2 \%)(p=0.387)$ for wtIDH and mutIDH patients, respectively.

\section{Positional analysis of recurrence}

The displacement of the rGTV centroid from the GTV and CTV centroids is outlined in Figure 4(b). The displacement of rGTV centroid for the wtIDH and mutIDH groups was $9.5 \pm 9.8 \mathrm{~mm}$ and $12.2 \pm 8.4 \mathrm{~mm}$ (p $=0.446)$ from the GTV and $14.6 \pm 8.8 \mathrm{~mm}$ and $15.9 \pm 7.9 \mathrm{~mm}(p=0.718)$ from the CTV, respectively.

The rGTV centroid was on average $1.4 \pm 6.7 \mathrm{~mm}$ and $0.7 \pm 1.5 \mathrm{~mm}(p=0.615)$ from the closest edge of the GTV for the wtIDH and mutIDH groups, respectively. As summarized in Figure 4(c), this distance was $0 \mathrm{~mm}$ (i.e. the rGTV centroid was within the GTV) for 21 (95\%) of the wtIDH patients; the sole patient with a non-zero distance had an rGTV centroid $31.4 \mathrm{~mm}$ from the closest portion of the GTV. This distance was also zero in 18 (82\%) mutIDH patients and ranged from $2.0-5.7 \mathrm{~mm}$ in the $4(18 \%)$ remaining mutIDH patients.

\section{Discussion}

Radiotherapy in glioblastoma and grade 4 IDH-mutant astrocytoma relies critically on the $1.5-3 \mathrm{~cm}$ expansion from the GTV to the CTV. An opportunity to further refine this margin relies on identifying patient subgroups for which this expansion could potentially be decreased. In this study, we demonstrated a differential recurrence pattern between these two groups with the results suggesting that the rGTV extends outside the planning GTV less often in the mutIDH group compared to wtIDH group. The volume of the recurrence extending beyond the GTV and CTV, however, is not statistically different between the two groups. In addition, there is no difference in terms of displacement of recurrent tumor centroid relative to the planning GTV or CTV.

IDH mutation is an increasingly important molecular biomarker of in terms of tumor behavior and therapy response in patients with high grade gliomas. In this study, we demonstrated that the GTV was larger in the mutIDH compared to wtIDH group. Unlike the wtIDH group, the tumor in mutIDH patients is thought to predominantly progress from lower grade gliomas [26] over a long time period and therefore had more 
time to slowly grow before causing clinical symptoms and thus being diagnosed. The larger decrease between the volume of the planning GTV and the recurrent tumor in the mutIDH group is also suggestive of better response to radiation in these patients. In turn, this may imply that interfraction variability is greater in the mutIDH group and patients with grade $4 \mathrm{IDH}$-mutant astrocytoma might therefore benefit from more frequent interfraction treatment assessment and adaptation [27-31]. An important caveat is that the patterns of recurrence analyzed here are based on standard radiotherapy volumes. While encouraging evidence exists that failure patterns are similar in the context of reduced margin radiotherapy [32,33], determination of the impact of margin reduction on patterns of recurrence is outside the scope of the study. With the recurrence volume smaller in the mutIDH group, and the centroid of the rGTV within $5.7 \mathrm{~mm}$ of the GTV for all patients in the mutIDH group, we believe that this patient group may particularly benefit from reduced margin radiotherapy. This is of special importance as these patients are younger compared to wtIDH group with longer overall survival. Margin reduction is a focus of ongoing studies which specify a $5 \mathrm{~mm}$ CTV, such as the Stanford hypofractionated stereotactic radiosurgery trial [34] and the UNITED study which employs daily MRI guidance and weekly adaptive replanning [35].

The results of this work demonstrated that the recurrent tumor extends less often beyond the planning GTV in the mutIDH group demonstrating the less aggressive biology of tumor in this group. Knowing that the main reason for contour expansion from GTV to CTV is to account for infiltrative microscopic disease, our results suggest that the current guidelines for expansion of GTV to CTV for radiation planning in glioblastoma patients $[11,12]$ can potentially be changed to be less conservative in grade 4 IDH-mutant astrocytoma. A definitive determination is, however, beyond the scope of this study and requires assessment in large prospective trails.

Our study also demonstrated that for those cases that the recurrence extended beyond the planning GTV and CTV, the recurrence volume extending beyond these radiotherapy planning structures was not statistically different between the mutIDH and wtIDH groups. That may indicate a subset of patients within the mutIDH group respond similarly to treatment compared to their wtIDH counterparts. Detailed molecular analysis or genomic sequencing data could potentially help define this subgroup; this data was, however, not available at the time of this study.

This study had several limitations. The retrospective nature of the study is a source of bias and the sample size in our study was small. Also, data regarding MGMT methylation status and other molecular biomarkers of high grade gliomas [36] were not available for this study. Large randomized trials are needed to draw conclusions that can potentiality further personalize radiation planning guidelines for glioblastoma based on patient specific molecular status.

\section{Declarations}

Acknowledgements: 
The authors gratefully acknowledge Sugantih Suppiah, Shadi Daghighi, Anish Kapadia, and Abdullah Alenezi for support with data collation, Hanbo Chen for statistical verification assistance, and Mark Ruschin for software development.

\section{Funding:}

Department of Radiation Oncology, Sunnybrook Odette Cancer Centre.

\section{Competing interests:}

Arjun Sahgal:

Advisor/consultant: AbbVie, Merck, Roche, Varian, Elekta, BrainLAB, and VieCure

Board member: International Stereotactic Radiosurgery Society (ISRS)

Co-Chair: AO Spine Knowledge Forum Tumor

Past educational seminars: Elekta, Accuray Inc., Varian, BrainLAB, Medtronic Kyphon

Research grants: Elekta

Travel accommodations/expenses: Elekta, Varian, BrainLAB

Membership: Elekta MR Linac Research Consortium, Elekta Spine, Oligometastases and Linac Based SRS Consortia

Jay Detsky:

Advisor/consultant: AbbVie, Bayer, Ferring

Sten Myrehaug:

Honoraria: AAA/Novartis, Ipsen

Research support: Ipsen

All other authors declare no competing interests

\section{Author Contributions:}

Conceptualization: AS, AKMC, PJM

Methodology \& Experimental Design: AS, AKMC, HS, CLT, JS, SM, JP, JK, MJLF, DM, GZ, DBS, PAL, PJM

Data Collection \& Collation: AKMC, HS, CLT, JD, SM, AH, CC 
Analysis: JS, AS, AKMC, PJM

Manuscript Writing: JS, AS, AKMC, PJM

Manuscript Review \& Editing: All authors

Manuscript Approval: All authors

\section{Availability of data and material:T}

he study data is available upon request from the corresponding authorpursuant to institutional, regulatory, and legal provisions.

\section{Code availability:}

Post processing codeis available upon request from the corresponding author pursuant to institutional, regulatory, and legal provisions.

\section{Ethics approval:}

This study was approved by the intuitional research ethics board with waiver of informed consent.

\section{Consent to participate:}

Informed consent was waived by the intuitional research ethics board.

\section{Consent for publication:}

Informed consent was waived by the intuitional research ethics board for publication of the data presented in this work.

\section{References}

1. Hartmann C, Hentschel B, Wick W, et al (2010) Patients with IDH1 wild type anaplastic astrocytomas exhibit worse prognosis than IDH1-mutated glioblastomas, and IDH1 mutation status accounts for the unfavorable prognostic effect of higher age: implications for classification of gliomas. Acta Neuropathol 120:707-718. https://doi.org/10.1007/s00401-010-0781-z

2. Songtao Q, Lei Y, Si G, et al (2012) IDH mutations predict longer survival and response to temozolomide in secondary glioblastoma. Cancer Sci 103:269-273. https://doi.org/10.1111/j.13497006.2011.02134.x

3. Louis DN, Perry A, Reifenberger · Guido, et al (2016) The 2016 World Health Organization Classification of Tumors of the Central Nervous System: a summary. Acta Neuropathol 131:803820. https://doi.org/10.1007/s00401-016-1545-1 
4. Louis DN, Perry A, Wesseling P, et al (2021) The 2021 WHO Classification of Tumors of the Central Nervous System: a summary. Neuro Oncol 23:1231-1251. https://doi.org/10.1093/neuonc/noab106

5. Eckel-Passow JE, Lachance DH, Molinaro AM, et al (2015) Glioma Groups Based on 1p/19q, IDH, and TERT Promoter Mutations in Tumors . N Engl J Med 372:2499-2508. https://doi.org/10.1056/nejmoa1407279

6. Verhaak RGW, Hoadley KA, Purdom E, et al (2010) Integrated Genomic Analysis Identifies Clinically Relevant Subtypes of Glioblastoma Characterized by Abnormalities in PDGFRA, IDH1, EGFR, and NF1. Cancer Cell 17:98-110. https://doi.org/10.1016/J.CCR.2009.12.020

7. Yan H, Parsons DW, Jin G, et al (2009) IDH1 and IDH2 Mutations in Gliomas. N Engl J Med 360:765773. https://doi.org/10.1056/nejmoa0808710

8. Combs SE, Rieken S, Wick W, et al (2011) Prognostic significance of IDH-1 and MGMT in patients with glioblastoma: One step forward, and one step back? Radiat Oncol 6:. https://doi.org/10.1186/1748-717x-6-115

9. Gravendeel LAM, Kouwenhoven MCM, Gevaert O, et al (2009) Intrinsic gene expression profiles of gliomas are a better predictor of survival than histology. Cancer Res 69:9065-9072. https://doi.org/10.1158/0008-5472.CAN-09-2307

10. Phillips HS, Kharbanda S, Chen R, et al (2006) Molecular subclasses of high-grade glioma predict prognosis, delineate a pattern of disease progression, and resemble stages in neurogenesis. Cancer Cell 9:157-173. https://doi.org/10.1016/j.ccr.2006.02.019

11. Stupp R, Mason WP, Van Den Bent MJ, et al (2005) Radiotherapy plus Concomitant and Adjuvant Temozolomide for Glioblastoma. N Engl J Med 352:987-996

12. Becker KP, Yu J (2012) Status quo-standard-of-care medical and radiation therapy for glioblastoma. Cancer J. 18:12-19

13. Burger PC, Dubois PJ, Schold SC, et al (1983) Computerized tomographic and pathologic studies of the untreated, quiescent, and recurrent glioblastoma multiforme. J Neurosurg 58:159-169. https://doi.org/10.3171/jns.1983.58.2.0159

14. Gaspar LE, Fisher BJ, Macdonald DR, et al (1992) Supratentorial malignant glioma: Patterns of recurrence and implications for external beam local treatment. Int J Radiat Oncol Biol Phys 24:5557. https://doi.org/10.1016/0360-3016(92)91021-E

15. Halperin EC, Bentel G, Heinz ER, Burger PC (1989) Radiation therapy treatment planning in supratentorial glioblastoma multiforme: An analysis based on post mortem topographic anatomy with ct correlations. Int J Radiat Oncol Biol Phys 17:1347-1350. https://doi.org/10.1016/03603016(89)90548-8

16. Chan JL, Lee SW, Fraass BA, et al (2002) Three-dimensional conformal radiotherapy. J Clin Oncol 20:1635-1642. https://doi.org/10.1016/s0169-5002(97)90162-8

17. Chang EL, Akyurek S, Avalos T, et al (2007) Evaluation of Peritumoral Edema in the Delineation of Radiotherapy Clinical Target Volumes for Glioblastoma. Int J Radiat Oncol 68:144-150. https://doi.org/10.1016/J.IJROBP.2006.12.009 
18. Mason WP, Del Maestro R, Eisenstat D, et al (2007) Canadian recommendations for the treatment of glioblastoma multiforme. Curr. Oncol. 14:110-117

19. Panet-Raymond V, Souhami L, Roberge D, et al (2009) Accelerated Hypofractionated IntensityModulated Radiotherapy With Concurrent and Adjuvant Temozolomide for Patients With Glioblastoma Multiforme: A Safety and Efficacy Analysis. Int J Radiat Oncol Biol Phys 73:473-478. https://doi.org/10.1016/j.ijrobp.2008.04.030

20. Dang L, White DW, Gross S, et al (2009) Cancer-associated IDH1 mutations produce 2hydroxyglutarate. Nature 462:739-744. https://doi.org/10.1038/nature08617

21. Ohgaki H, Kleihues $P$ (2013) The definition of primary and secondary glioblastoma. Clin. Cancer Res. 19:764-772

22. Wen PY, Macdonald DR, Reardon DA, et al (2010) Updated response assessment criteria for highgrade gliomas: Response assessment in neuro-oncology working group. J. Clin. Oncol. 28:19631972

23. Niyazi M, Brada M, Chalmers AJ, et al (2016) ESTRO-ACROP guideline "target delineation of glioblastomas." Radiother Oncol 118:. https://doi.org/10.1016/j.radonc.2015.12.003

24. Tseng CL, Stewart J, Whitfield G, et al (2020) Glioma consensus contouring recommendations from a MR-Linac International Consortium Research Group and evaluation of a CT-MRI and MRI-only workflow. J Neurooncol 149:305-314. https://doi.org/10.1007/s11060-020-03605-6

25. Yushkevich PA, Piven J, Hazlett HC, et al (2006) User-guided 3D active contour segmentation of anatomical structures: Significantly improved efficiency and reliability. Neuroimage 31:1116-1128. https://doi.org/10.1016/j.neuroimage.2006.01.015

26. Ichimura K, Pearson DM, Kocialkowski S, et al (2009) IDH1 mutations are present in the majority of common adult gliomas but rare in primary glioblastomas. Neuro Oncol 11:341-347. https://doi.org/10.1215/15228517-2009-025

27. Lee S, Stewart J, Lee Y, et al (2019) Improved dosimetric accuracy with semi-automatic contour propagation of organs-at-risk in glioblastoma patients undergoing chemoradiation. J Appl Clin Med Phys 20:45-53. https://doi.org/10.1002/acm2.12758

28. Manon R, Hui S, Chinnaiyan P, et al (2004) The impact of mid-treatment MRI on defining boost volumes in the radiation treatment of glioblastoma multiforme. Technol Cancer Res Treat 3:303307. https://doi.org/10.1177/153303460400300308

29. Tsien C, Gomez-Hassan D, Ten Haken RK, et al (2005) Evaluating changes in tumor volume using magnetic resonance imaging during the course of radiotherapy treatment of high-grade gliomas: Implications for conformal dose-escalation studies. Int J Radiat Oncol Biol Phys 62:328-332. https://doi.org/10.1016/j.ijrobp.2004.10.010

30. Kim TG, Lim DH (2013) Interfractional variation of radiation target and adaptive radiotherapy for totally resected glioblastoma. J Korean Med Sci 28:1233-1237. https://doi.org/10.3346/jkms.2013.28.8.1233 
31. Stewart J, Sahgal A, Lee Y, et al (2021) Quantitating Interfraction Target Dynamics During Concurrent Chemoradiation for Glioblastoma: A Prospective Serial Imaging Study. Int J Radiat Oncol Biol Phys 109:736-746. https://doi.org/10.1016/j.ijrobp.2020.10.002

32. Paulsson AK, McMullen KP, Peiffer AM, et al (2014) Limited margins using modern radiotherapy techniques does not increase marginal failure rate of glioblastoma. Am J Clin Oncol Cancer Clin Trials 37:177-181. https://doi.org/10.1097/COC.0b013e318271ae03

33. McDonald MW, Shu HKG, Curran WJ, Crocker IR (2011) Pattern of failure after limited margin radiotherapy and temozolomide for glioblastoma. Int J Radiat Oncol Biol Phys 79:130-136. https://doi.org/10.1016/j.ijrobp.2009.10.048

34. Azoulay M, Chang SD, Gibbs IC, et al (2020) A phase I/II trial of 5-fraction stereotactic radiosurgery with 5-mm margins with concurrent temozolomide in newly diagnosed glioblastoma: Primary outcomes. Neuro Oncol 22:1182-1189. https://doi.org/10.1093/neuonc/noaa019

35. Detsky J (2021) NCT04726397 - UNIty-Based MR-Linac Guided AdapTive RadiothErapy for High GraDe Glioma: A Phase 2 Trial (UNITED). In: ClinicalTrials.gov

36. Zhang P, Xia Q, Liu L, et al (2020) Current Opinion on Molecular Characterization for GBM Classification in Guiding Clinical Diagnosis, Prognosis, and Therapy. Front. Mol. Biosci. 7

\section{Figures}

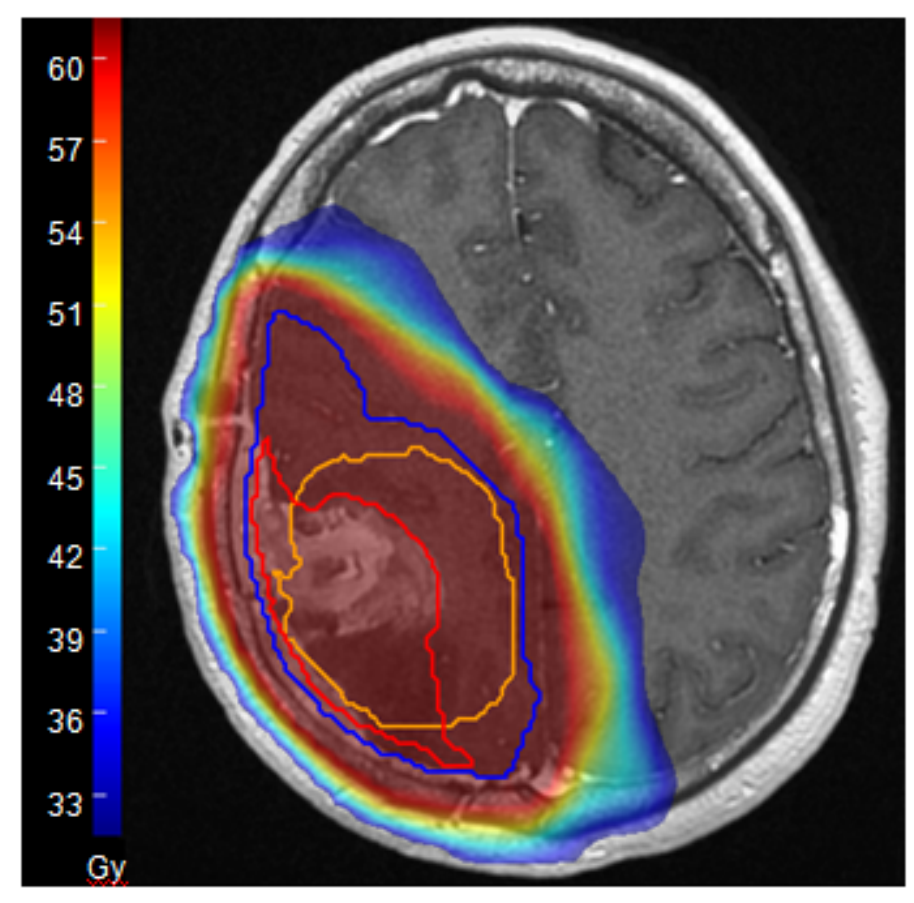

(a)

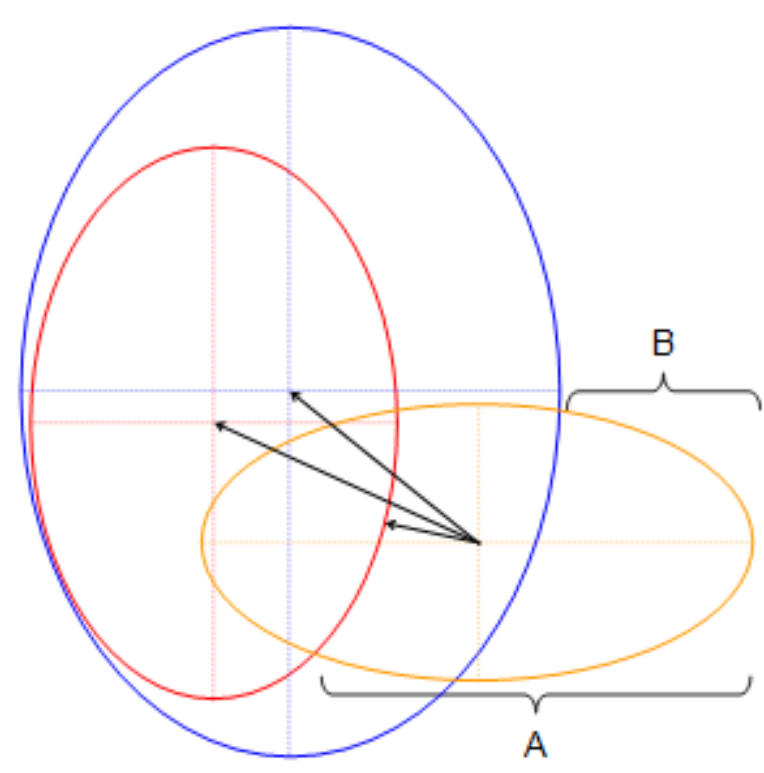

(b)

\section{Figure 1}

(a) Axial slice of patient with grade 4 IDH-mutant astrocytoma illustrating analysis volumes. Overlaid on the T1 with Gadolinium contrast planning MRI are the gross tumor volume (GTV; red contour), clinical 
target volume (CTV; blue contour), and recurrent gross tumor volume (rGTV; orange contour). Also shown for illustrative purposes is the 60 Gy prescribed radiotherapy dose plan (colorwash). (b) Schematic twodimensional illustration of metrics quantified in this study. The GTV, CTV, and rGTV are denoted by the red, blue, and orange contours, respectively; the centroid of each contour is indicated. The three arrows demonstrate the distance of the rGTV centroid from the GTV centroid, CTV centroid, and closest edge of the GTV. The regions of the rGTV outside the GTV (labelled 'A') and CTV ('B') are also indicated.

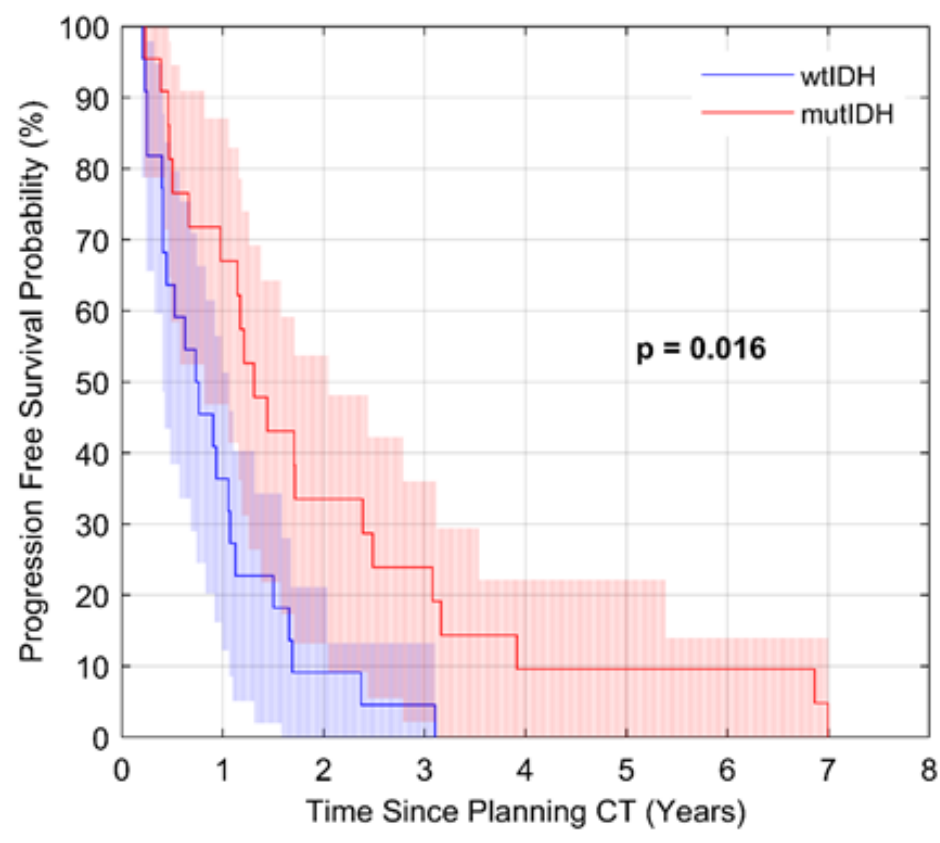

(a)

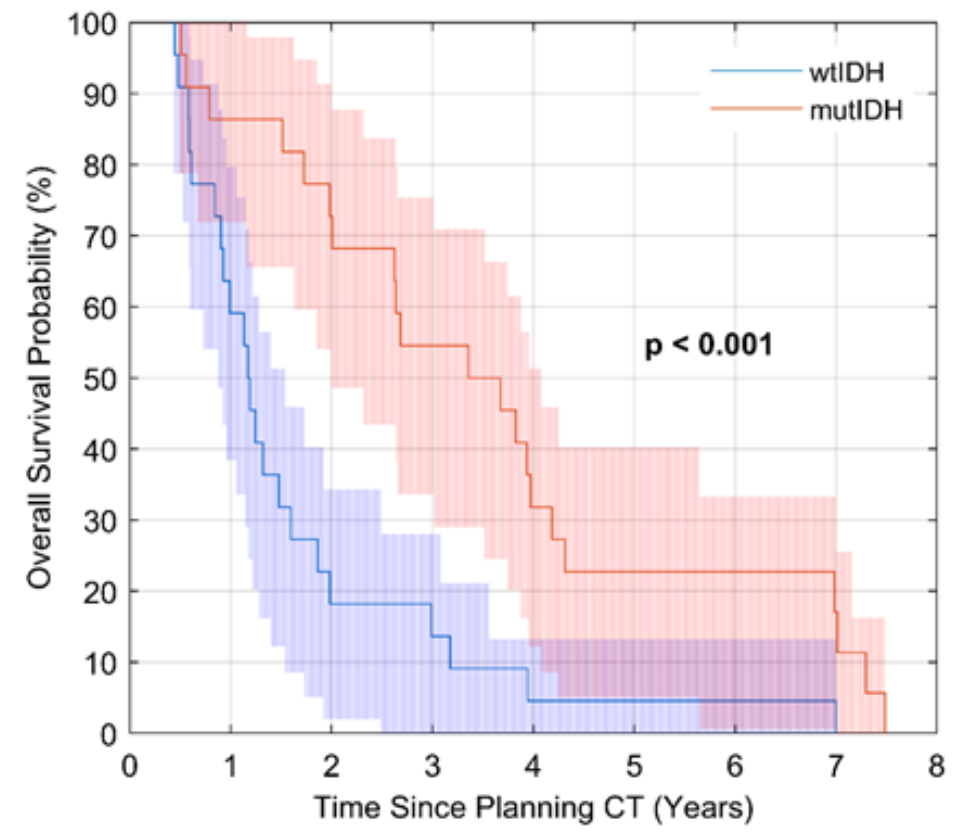

(b)

\section{Figure 2}

Kaplan-Meier estimates of progression free survival (a) and overall survival (b) for the wtIDH (blue) and mutIDH (red) groups with 95\% confidence interval delineated by the shaded region. 


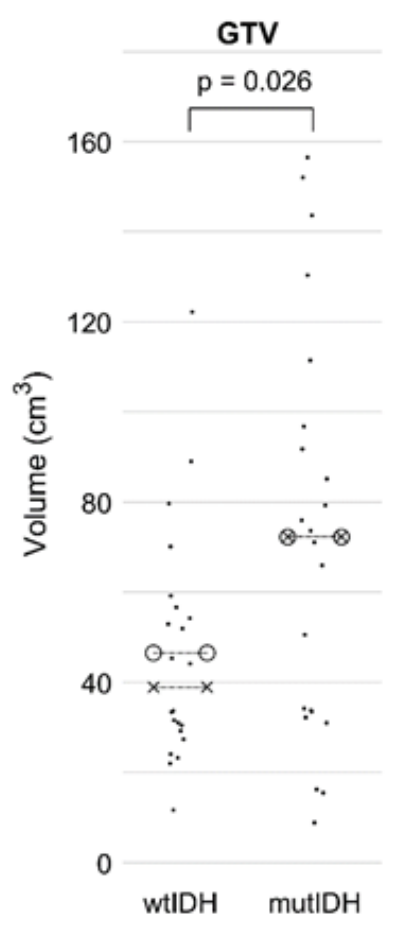

(a)
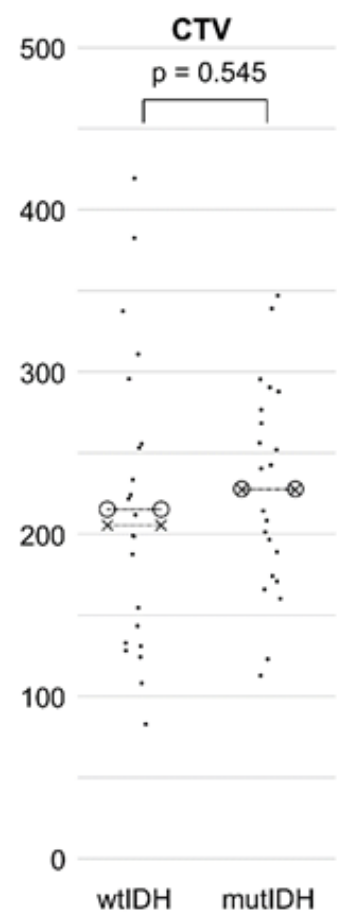

(b)
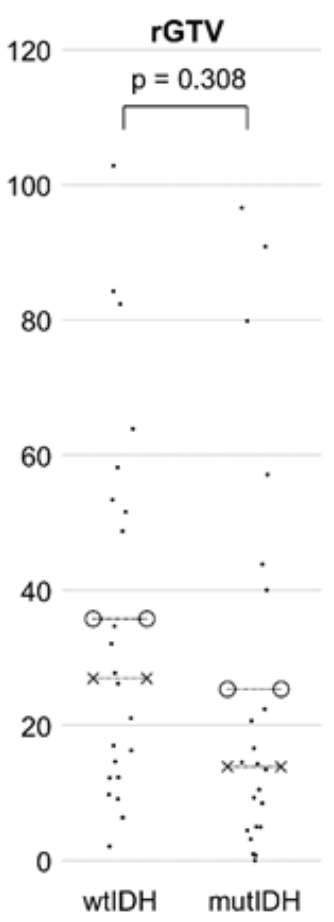

(c)

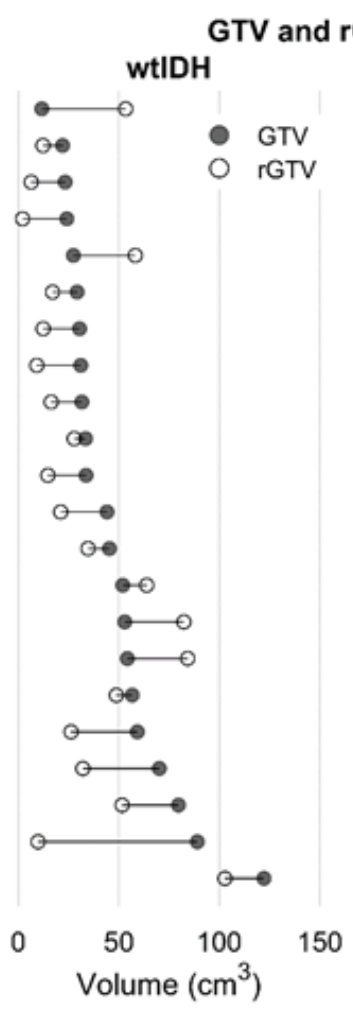

(d)

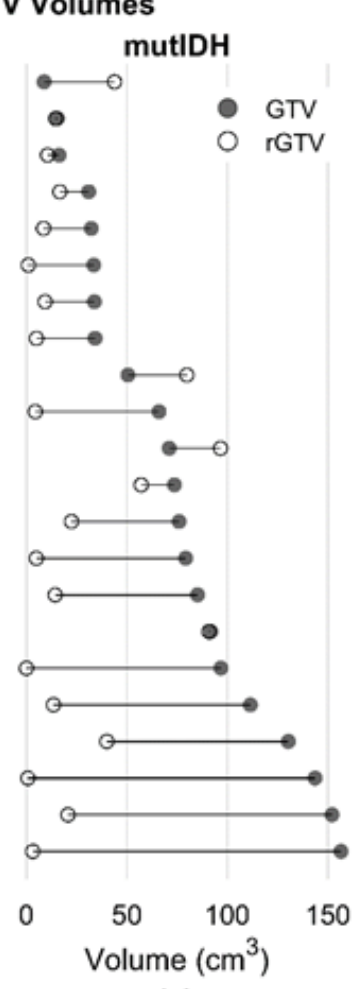

(e)

\section{Figure 3}

(a)-(c) Gross tumor (GTV), clinical targeting (CTV), and recurrent gross tumor (rGTV) volumes for the wtIDH and mutIDH groups. In all three panels, the scatter points denote individual patient volumes; points are randomly offset along the abscissa to improve visualization. The horizontal dashed lines with 'o' and ' $x$ ' endpoints denote the mean and median, respectively, of the group. The GTV and rGTV volume data are delineated in a different form in panels (d)-(e) to highlight individual patient differences between these two volumes. In both panels, the $n=22$ patients in each of the wtIDH (d) and mutIDH (e) groups are shown along the ordinal with the filled and open circles denoting the GTV and rGTV volumes, respectively. Patients are sorted by their GTV volume. The rGTV was $10.7 \pm 26.9 \mathrm{~cm} 3$ (mean \pm SD) and $46.9 \pm 55.0$ cm3 smaller than the GTV for wtIDH and mutIDH groups, respectively $(p=0.018)$. 


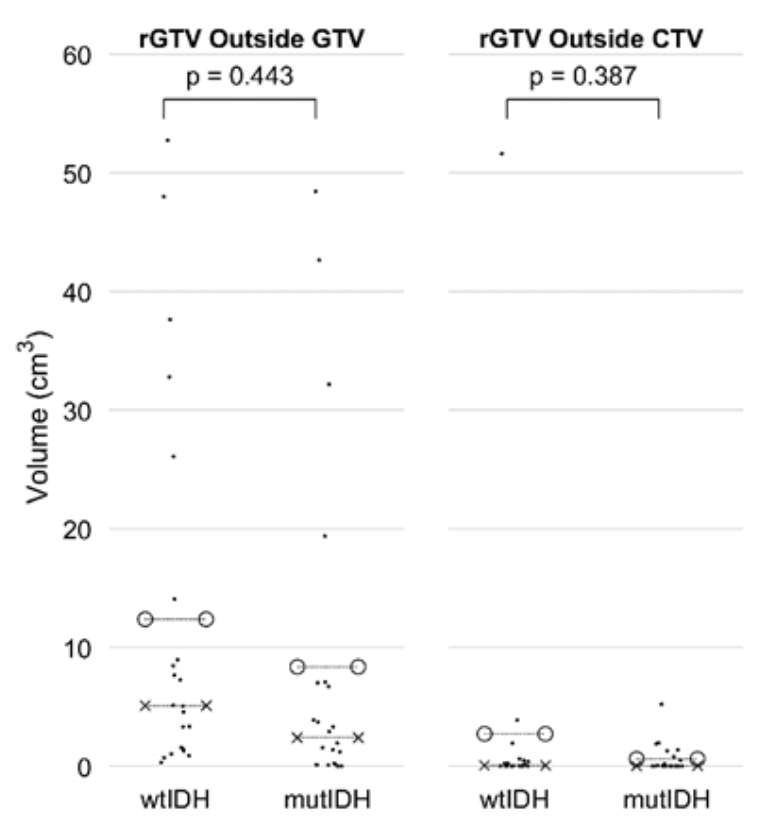

(a)

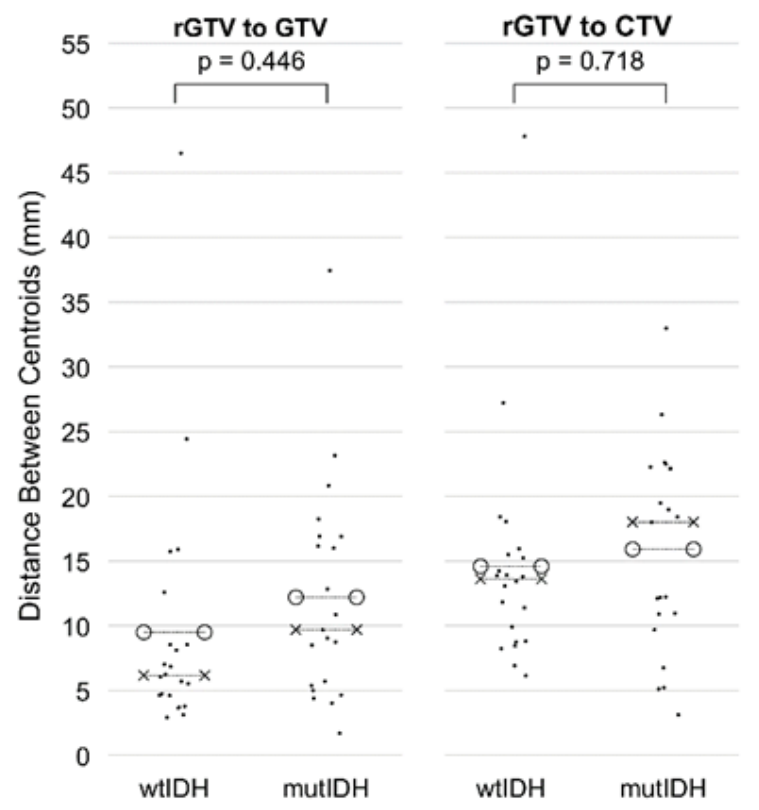

(b)

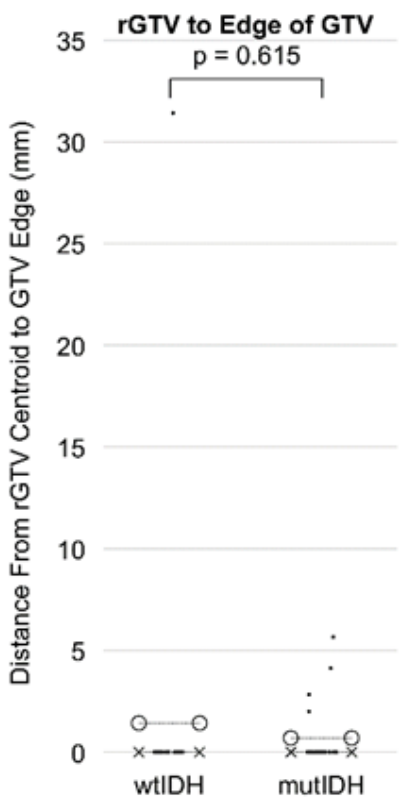

(c)

\section{Figure 4}

Volumetric (a) and positional (b-c) analyses of recurrent gross tumor volume (rGTV) for the wtIDH and mutIDH groups. (a) rGTV volume outside the gross tumor volume (GTV; left panel) and clinical target volume (CTV; right panel). (b) Distance of the rGTV centroid from the GTV (left panel) and CTV (right panel) centroids. (c) Distance of the rGTV centroid from the closest edge of the GTV. In all panel groups in the scatter points denote individual patient measurements; these points are randomly offset along the abscissa to improve visualization. The horizontal dashed lines with ' 0 ' and ' $x$ ' endpoints denote the mean and median, respectively, of the group. 